\title{
Antidiabetic activity of cobalt-quercetin complex: A new potential candidate for diabetes treatment
}

\author{
Mohamed M. Hassanien ${ }^{1}$, Entsar A. Saad ${ }^{2 *}$, Kholoud H. Radwan ${ }^{3}$ \\ ${ }^{1}$ Chemistry Department, Faculty of Technology and Education, Beni-Suef University, Beni-Suef, Egypt. \\ ${ }^{2}$ Chemistry Department, Faculty of Science, Damietta University, Damietta, Egypt. \\ ${ }^{3}$ Department of Biochemistry, Horus University in Egypt HUE, Damietta, Egypt.
}

\begin{tabular}{l}
\hline ARTICLE INFO \\
\hline Received on: 06/07/2020 \\
Accepted on: $28 / 10 / 2020$ \\
Available online: 05/12/2020 \\
\\
\hline Key words: \\
Antidiabetic, antioxidant, \\
cobalt-quercetin complex, \\
defensive, hypolipidemic.
\end{tabular}

\section{INTRODUCTION}

Diabetes mellitus (DM) is a chief endocrine disease and expanding health issue in almost all countries. It is one of the top five illnesses causing early death worldwide. Nearly 171 million individuals in all countries were diagnosed with diabetes in 2000 and by the year 2030, it is expected that 366 million people will have the disease (Wild et al., 2004). In Egypt, DM is the foremost prominent medical and public health challenge. The overall measure of diabetic individuals in Egypt will elevate from 3.80 million in 2000 to 30 million by the year 2025 (Badran et al., 1997).

\footnotetext{
*Corresponding Author

Entsar A. Saad, Chemistry Department, Faculty of Science, Damietta University,Damietta,Egypt.E-mail: entsarsaad@gmail.com
}

DM is featured with insufficiency of insulin release and/ or action, insulin resistance, and abnormal glucose, lipid, and protein pathways. It causes multiple health consequences like kidney impairment, vision loss, and leg removal, and may even cause early death (Saad et al., 2019a; Shaikh and Shrivastava, 2014). Although we have a number of oral hypoglycemic agents alongside insulin to cure DM, no one introduces perfect glycemic adjustment (Shaikh and Shrivastava, 2014). Hence, there is a continual demand to explore new efficient antidiabetic medications.

In fact, oxidative stress plays a crucial role in many pathological processes, such as obesity, cardiovascular diseases (Habib et al., 2015), liver diseases (Attallah et al., 2015; Saad et al., 2015a; Saad et al., 2017a), renal diseases (Habib et al., 2020; Saad et al., 2018a, 2018b), cancer (Saad et al., 2020a, 2020b), and diabetes (Saad et al., 2017b). Other research studies have confirmed that the advance of diabetic-induced consequences in DM is closely linked with the high production of superoxide anion 
and NO (Abou-seif and Youssef, 2004). Oxygen/nitrogen-reactive molecules are produced excessively in DM through oxidative breakdown of glucose, enzyme-independent glycation of proteins, and oxygen-dependent breakdown of glycated-proteins (Mehta et al., 2006). Abnormal excessive levels of reactive species and the concomitant drop in internal antioxidant defense lines may result in damaged cellular components, high lipid peroxidation rate (El-Aassar et al., 2019; Elsayed et al., 2019), and insulin resistance (Naggar et al., 2005). These oxidative stress sequels may support diabetes consequences advance inspiring a novel and innovative strategy to a potential "causal" antioxidant remedies, for example, the flavonoids.

Flavonoids $(>8,000$ are known) are the greatest and most significant set of plant polyphenols. They are extensively spread in multiple frequently consumed drinks and foodstuff of plant origin (Ross and Kasum, 2002). Some examples of dietary flavonoids are catechin, epicatechin, quercetin, and rutin in red grapes (Iacopini et al., 2008), curcumin in the Indian spice turmeric (Patil et al., 2017), and quercetin in almost all edible vegetables and fruits (Zahran et al., 2019). Accordingly, the development of effective, available, safe, and less costly antidiabetic synthetic preparations based on flavonoids is of interest.

Dietary flavonoids are demonstrated to be powerful antioxidants; they inhibit the formation of active-oxygen/nitrogen radicals and affect the activities of many detoxifying catalytic molecules (Zahran et al., 2019). Quercetin $\left(3,3^{\prime}, 4^{\prime}, 5,7\right.$ pentahydroxy flavone), as a member of the dietary flavonoids, has a potent antioxidant activity and could significantly participate in body protection versus long-lasting illnesses. Quercetin is characterized by a broad scope of bioactivities, for example, anti-inflammatory, antioxidant, antitumor, immunomodulatory, antiulcer, and vasodilator effects (Shoskes and Nickel, 2011).

Transition metal ions are present in different living organisms and are noted to play essential roles in diverse enzyme-dependent and functional reactions. They give a hand in the starting of the reactive species process through the Fenton reaction (Bukhari et al., 2008). An example of this is cobalt, which is fundamental and highly required for good body health, since it aids in nutrients digestion, conserves the metabolic system very potent, and protects the body against bacteria and viruses. Pharmacologically, cobalt complexes are worth taking into account due to their antibacterial, antifungal, and antitumor bioactivities (Chang et al., 2010; Saad et al., 2017c).

Therefore, the cobalt-quercetin complex (CQC), previously synthesized and well characterized by Bukhari et al. (2008), was selectively chosen by us for this study for many reasons. First, $\mathrm{CQC}$ is based on the well-known natural product quercetin and one of our body transition elements, cobalt, so high safety and compatibility are expected. Second, it is characterized by water-solubility, low-cost, and easy CQC preparation. CQC can be easily freshly prepared in an aqueous solution whenever needed simply by just mixing the solution of two preprepared (quercetin and cobalt chloride) working reagents that are commercially available at a reasonable price. Third, $\mathrm{CQC}$ has in vitro antioxidant activity. Finally, and above all, CQC has not been investigated before for the current study purpose. All these reasons encouraged us to investigate its antidiabetic, hypolipidemic, antioxidant, and defensive effects on vital organs (pancreas, liver, and kidney) in comparison to insulin (as a reference drug) in induced IDDM rats. The effects of CQC administration on healthy normal rats were also studied to judge its safety.

\section{MATERIALS AND METHODS}

\section{Chemicals, drugs, and kits}

Chemical reagents with the highest accessible degree of purity were used. Solvents were redistilled by standard methods before usage. Quercetin and streptozotocin (STZ) (Sigma, St. Louis, MO), $\mathrm{CoCl}_{2} \cdot 6 \mathrm{H}_{2} \mathrm{O}$ (Riedel-de Haen, Seelze, Germany), zinc-insulin suspension (Lente insulin) (Monotard, Novo Nordisk, Bagsvaerd, Denmark), creatinine and urea kits (Diamond Diagnostics, Holliston, MA), aspartate aminotransferase (AST) and alanine aminotransferase (ALT) kits (ELITech Clinical Systems, Sées, France), albumin kit (STANBIO Company, Boerne, TX), uric acid, total cholesterol, high-density lipoprotein (HDL)-cholesterol and triglycerides (TG) kits (SPINREACT, Santa Coloma, Sant Esteve De Bas, Spain), and MDA, superoxide dismutase (SOD), nitric oxide (NO), and total antioxidant capacity (TAC) kits (Biodiagnostic Company, Cairo, Egypt) were used.

\section{In-solution synthesis and elucidation of CQC}

Quercetin was recrystallized twice in isopropanol and then its purity was checked by getting one spot using thin layer chromatography (TLC). $\mathrm{CoCl}_{2} \cdot 6 \mathrm{H}_{2} \mathrm{O}$ solution $0.1 \mathrm{mM}$ was well standardized using complexometry standardized method. To prepare CQC, a continuous variation procedure was employed to verify the stoichiometric composition of the chelate spectrophotometrically, as described by Saad et al. (2017d). The absorbance visible peak of quercetin at $372 \mathrm{~nm}$ was decreased and a novel distinctive peak of the complex was detected at 427 $\mathrm{nm}$ with adding $\mathrm{CoCl}_{2} .6 \mathrm{H}_{2} \mathrm{O}$ solution. The absorbance plot at 427 $\mathrm{nm}$ versus the molar fraction of quercetin $(\mathrm{X})$ displayed maximal absorbance at $X_{\mathrm{L}}=0.33$ (Fig. 1), approving that the stoichiometric ratio is $2: 1$. Then, the $\mathrm{CQC}$ was prepared by mixing one volume of quercetin solution $(0.1 \mathrm{mM}$ in absolute ethanol) with two volumes of $\mathrm{CoCl}_{2} \cdot 6 \mathrm{H}_{2} \mathrm{O}$ solution $(0.1 \mathrm{mM}$ in bidistilled water).

\section{Determination of CQC median lethal dose $\left(\mathrm{LD}_{50}\right)$}

The $\mathrm{LD}_{50}$ was estimated as previously represented in Saad et al. (2017e). The CQC solution was administered to four groups of Swiss albino rats (four rats/group) at different dosages $\left(25,50,100\right.$, and $\left.150 \mathrm{mg} \mathrm{Co}{ }^{2+} / \mathrm{kg}\right) . \mathrm{LD}_{50}$ was evaluated by recording mortality after 72 hours. $\mathrm{LD}_{50}$ of the complex was 62.5 $\mathrm{mg} \mathrm{Co}{ }^{2+} / \mathrm{kg}$ and a dose of $42 \%$ of the $\mathrm{LD}_{50}$ was selected for animal treatment.

\section{Maintenance of animals}

Adult male Swiss albino rats weighing 130-165 g bought from the Urology and Nephrology Centre, Mansoura University, Egypt, were utilized. All animals were housed for about 10 days before use in the experiment under a standard environment (temperature of $25^{\circ} \mathrm{C}$, relative humidity, and a $12 / 12$ hours light/ dark cycle) as stated by the standards defined in the "Guide for the Care and Use of Laboratory Animals" designed by the National Academy of Sciences and published by the National Institute of Health. Standard chow and water ad libitum were permitted. This 


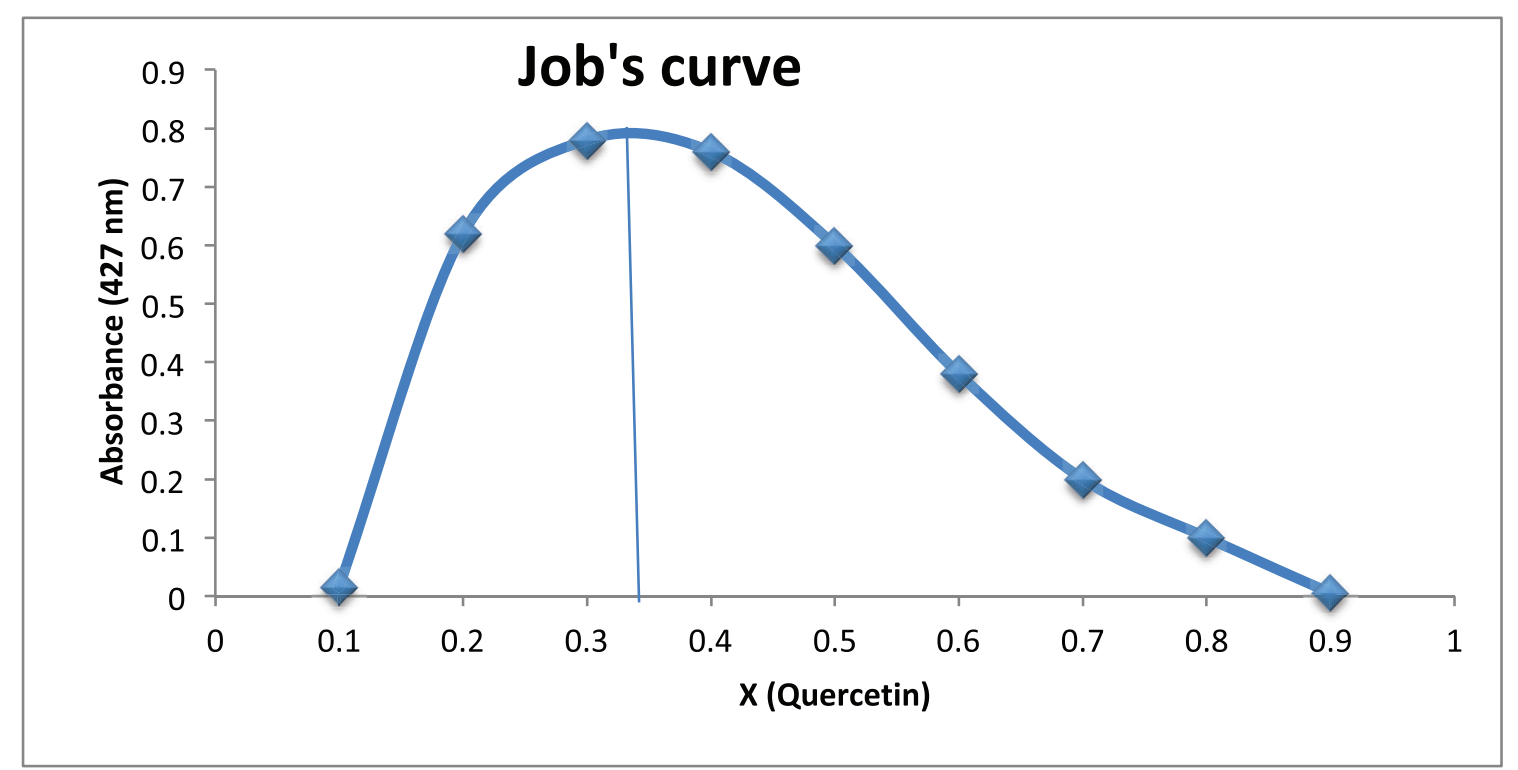

Figure 1. Continuous variation method. $427 \mathrm{~nm}$ is $\lambda_{\max }$ of chelate and $\mathrm{X}$ is the mole fraction of quercetin.

was approved by the Animal House of Biochemistry, Chemistry Department, Faculty of Science, Damietta University, Egypt.

\section{Induction of DM}

The rats were fasted for 12 hours and DM was prompted via a single intravenous injection of freshly prepared STZ at a dosage of $50 \mathrm{mg} / \mathrm{kg}$ bodyweight. STZ was dissolved in ice-cold sodium citrate buffer (0.1 M, pH 4.5) (Saad et al., 2015b). Normal control rats received a corresponding volume of buffer intravenously. Rats were permitted to drink glucose $(5 \%)$ overnight to cope with druginduced hypoglycemia. Diabetes was assured after 48 hours of STZ administration via estimating fasting blood glucose (FBG) levels from tail vein employing commercial glucose strips and a glucometer from Boehringer-Mannheim Diagnostics, Mannheim, Germany. Only animals with FBG concentration of $250 \mathrm{mg} / \mathrm{dl}$ and above were regarded as diabetic and were used for the experiment. The day on which diabetes had been confirmed was designated as day zero.

\section{Experimental design}

Animals were divided into five groups (seven rats/group) as follows: normal control group involved rats of no treatment; normal + CQC group involved normal rats treated with a daily oral dose of CQC $\left(42 \% \mathrm{LD}_{50}\right)$; diabetic control group involved rats which received one dosage of STZ (50 mg/kg, IV); diabetic + insulin group which involved STZ-induced diabetic rats (50 $\mathrm{mg} / \mathrm{kg}, \mathrm{IV})$ treated with the standard drug insulin (6 IU/kg, SC) once every 48 hours for 15 days; and diabetic + CQC group which involved STZ-induced diabetic animals $(50 \mathrm{mg} / \mathrm{kg}$, IV) treated with a daily oral dose of CQC $\left(42 \% \mathrm{LD}_{50}\right)$ through a stomach tube for 15 days.

The food and water intakes of all groups were recorded daily, while bodyweights and FBG levels were determined weekly throughout the experimental period.

\section{Samples}

On the 16th day and after fasting for 12 hours, all rats were sacrificed under diethyl ether anesthesia and blood samples were withdrawn via the retroorbital venous plexus. Only $0.1 \mathrm{ml}$ of each sample was used for the determination of glycosylated hemoglobin A1C (HbA1C). The rest of each whole blood sample was settled to coagulate and the serum was separated by centrifugation $\left(4^{\circ} \mathrm{C}, 3,000 \mathrm{rpm}, 10\right.$ minutes $)$ and kept at $-20^{\circ} \mathrm{C}$ till utilized for biochemical estimations.

\section{Biochemical measurements}

All measurements of the biochemical parameters were carried out according to the kits' instructions.

\section{Estimation of $\mathrm{HbA1C}$}

Separation and identification of $\mathrm{HbA} 1 \mathrm{C}$ were done using cation exchange chromatographic techniques (Schellekens et al., 1981).

\section{Histopathological analysis}

The liver and pancreas excised after death were cleaned up using $\mathrm{NaCl}$ solution and then fixed in formalin (10\%) to be used for the classical hematoxylin and eosin (H\&E) staining system. Fixed tissues were implanted in paraffin, cut into $5 \mu \mathrm{m}$ thick segments, and then stained using H\&E dye. The stained sections of three or more samples were blindly subjected to examination and evaluation by an expert pathologist.

\section{Statistical analysis}

The obtained data are introduced as mean $\pm \mathrm{SD}$. Student's $t$-test was employed for analysis between two groups. $p<0.05$ was designated as significant. All the analyses were achieved using InStat Version 3.10 (GraphPad, San Diego, CA). 


\section{RESULTS}

\section{Water and food intakes}

Our results showed that, in normal rats treated with CQC, a significant rise ( $p<0.001 v s$. normal control) in water consumption was observed from day 5 and continued to the last day of treatment, accompanied by a significant decrease $(p<0.001$ $v s$. normal control) in food intake noted from day 5 that was turned back to increase on day 15 . In addition, the volume of water intake in the diabetic control group was greater $(p<0.001)$ than that in the normal control group and increased progressively till the experiment was terminated, while in diabetic rats supplemented with insulin or CQC, significant decreases $(p<0.001 v s$. diabetic control) in the water intake were observed from day 10; however, the values were still higher than those prior to the induction of diabetes. Moreover, food intake was declined $(p<0.001 v s$. normal control) in the diabetic control group from day 5 and proceeded till the end of the experiment, and it was significantly ( $p<0.001 \mathrm{vs}$. diabetic control) and continuously increased in the treated diabetic rats with either insulin or CQC from day 10 till the experiment was terminated (Table 1).

\section{Bodyweight}

There was an obvious reduction in the bodyweight of animals in the diabetic group in comparison to the control group animals. After the treatment of diabetic rats with CQC, the bodyweight continued to rise, during the second week and continued, and it was notably regained to around normal level, at the end of the experiment. In the case of treatment of diabetic animals with insulin, the rats began later to have a bodyweight increase at the end of the experiment and this increase was to a lesser extent. On the other hand, a marked higher elevation in the bodyweight of normal control group rats administered with CQC was noted from day 14 and continued (Table 2).

\section{FBG level}

In either insulin- or CQC-treated diabetic rats, hypoglycemic effects were evident $(p<0.001)$ from day 7 onwards the decreases in blood glucose levels were highly pronounced on day 14 . On the other hand, in the normal rat group treated with CQC, there were no significant changes $(p>0.05)$, which were observed from the first day to the last day of the experiment in the FBG levels when compared with those of the corresponding normal control rats group (Table 2).

\section{Biochemical parameters}

Blood glycosylated HbAlC

Table 3 shows that the normal group treated with CQC showed no significant changes in blood $\mathrm{HbA} 1 \mathrm{C}$ when compared with the normal control group. Treatment with insulin or CQC reversed high diabetic-induced blood $\mathrm{HbA} 1 \mathrm{C}$ levels to normal.

Table 1. Water and food intakes at different intervals of rats' groups.

\begin{tabular}{|c|c|c|c|c|c|}
\hline Groups & Normal control & Normal + CQC & Diabetic control & Diabetic + insulin & Diabetic + CQC \\
\hline \multicolumn{6}{|c|}{ Water intake (ml/group/day) } \\
\hline Day 5 & $123 \pm 3.109$ & $152.7 \pm 2.217^{* * *}$ & $211 \pm 2.709^{* * * *}$ & $201 \pm 10.033$ & $219.0 \pm 2.602$ \\
\hline Day 10 & $120 \pm 4.349$ & $142 \pm 2.082^{* * *}$ & $276.5 \pm 5.066^{* * *}$ & $192.25 \pm 2.217^{\$ \$ \$}$ & $208.0 \pm 5.066^{\mathrm{ss}}$ \\
\hline Day 15 & $122.2 \pm 1.708$ & $143.5 \pm 1.291^{* * * *}$ & $282.25 \pm 2.217^{* * * *}$ & $184.5 \pm 4.203^{\text {s\$s }}$ & $190.0 \pm 2.203^{s s s}$ \\
\hline \multicolumn{6}{|c|}{ Food intake (g/group/day) } \\
\hline Day 5 & $300.25 \pm 2.449$ & $272.5 \pm 5.260^{* * * *}$ & $262 \pm 2.449^{* * *}$ & $259.25 \pm 3.304$ & $203.25 \pm 2.500^{s s s}$ \\
\hline Day 10 & $305.5 \pm 3.697$ & $270.5 \pm 3.109^{* * * *}$ & $242 \pm 2.082^{* * *}$ & $267.25 \pm 2.630^{\$ \$ \$}$ & $214 \pm 4.546^{\$ \$ s}$ \\
\hline Day 15 & $314.25 \pm 4.349$ & $276.25 \pm 2.630^{* * * *}$ & $235 \pm 3.559^{* * * *}$ & $268.25 \pm 4.573^{\$ \$ \$}$ & $221 \pm 2.160^{\$ s \$}$ \\
\hline
\end{tabular}

Values are introduced as mean $\pm \mathrm{SD}(n=7$ rats/group). CQC: cobalt-quercetin complex.

${ }^{* * * *} P<0.001$ against normal group.

${ }^{\$ \$ \$} P<0.001$ against diabetic group.

Table 2. Bodyweight and FBG $(\mathrm{mg} / \mathrm{dL})$ in different intervals of rats' groups.

\begin{tabular}{|c|c|c|c|c|c|}
\hline Groups & Normal control & Normal + CQC & Diabetic control & Diabetic + insulin & Diabetic + CQC \\
\hline \multicolumn{6}{|c|}{ Bodyweight (g/rat) } \\
\hline Day 0 & $151.78 \pm 1.58$ & $167.0 \pm 3.750$ & $160.0 \pm 9.126$ & $187.8 \pm 9.850$ & $153.4 \pm 2.11$ \\
\hline Day 7 & $160.9 \pm 3.740$ & $163.6 \pm 2.120$ & $149.0 \pm 5.660$ & $162.0 \pm 3.090$ & $139.1 \pm 3.804$ \\
\hline Day 14 & $169.7 \pm 2.04$ & $177.4 \pm 3.150$ & $137.0 \pm 1.530$ & $166.4 \pm 3.625$ & $151.8 \pm 3.07$ \\
\hline \multicolumn{6}{|c|}{$\mathrm{FBG}(\mathrm{mg} / \mathrm{dl})$} \\
\hline Day 0 & $109.0 \pm 3.26$ & $114.6 \pm 7.091$ & $303.0 \pm 21.71^{* * * *}$ & $265.2 \pm 14.410^{* * * *}$ & $272.1 \pm 10.029^{* * * *}$ \\
\hline Day 7 & $113.5 \pm 3.99$ & $116.0 \pm 5.460$ & $317.0 \pm 11.04^{* * *}$ & $185.8 \pm 22.790^{\$ \$ \$}$ & $220.0 \pm 20.81^{\$ \$ \$}$ \\
\hline Day 14 & $123.1 \pm 3.44$ & $118.57 \pm 6.901$ & $322.0 \pm 11.20^{* * * *}$ & $162.5 \pm 17.855^{\$ \$}$ & $159.5 \pm 3.82^{\$ s \$}$ \\
\hline
\end{tabular}

Values are introduced as mean $\pm \mathrm{SD}(n=7$ rats/group). CQC: cobalt-quercetin complex.

${ }^{* * * *} P<0.001$ against normal group.

${ }^{\text {s\$ }} P<0.001$ against diabetic group. 


\section{Liver function tests}

As indicated in Table 3, serum AST and ALT activities were significantly $(p<0.001)$ inhibited while serum albumin level was highly increased $(p<0.001)$ by insulin or CQC supplementation when compared with those of the diabetic control group. Furthermore, a significant $(p<0.001)$ increase in the activity of both AST and ALT along with a significant decrease $(p<0.001)$ in serum albumin was detected in the normal group treated with CQC when compared with the untreated normal control group.

\section{Kidney function tests}

As presented in Table 3, STZ administration showed significant $(p<0.001)$ elevations in circulated concentrations of uric acid, urea, and creatinine. Treatment of diabetic rats with insulin significantly reduced uric acid, creatinine, and urea levels ( $p<0.01, p<0.001$, and $p<0.001$, resp.) compared to those of the nontreated diabetic group. CQC supplementation to diabetic rats significantly diminished creatinine, uric acid, and urea concentrations ( $p<0.05, p<0.01$, and $p<0.001$, resp.) compared to those of the nontreated diabetic group. Moreover, CQC supplementation to normal rats kept serum creatinine, urea, and uric acid levels within their normal ranges when compared with normal rats' group levels.

\section{Lipid profile}

Significant decreases in serum levels of cholesterol, lowdensity lipoprotein (LDL), and TG accompanied by a significant elevation in serum HDL level were noted in diabetic rats administered with insulin or CQC compared to those of untreated diabetic rats. In addition, normal rats administered with CQC showed normal lipid profile except a slight significant $(p<0.05)$ elevation in TG level which was detected (Table 3 ).

\section{Lipid peroxidation and antioxidants}

Table 3 illustrates a significant $(p<0.001)$ elevation, to more than sixfold, in serum lipid peroxidation marker (MDA) and a significant $(p<0.001)$ elevation in NO concentration along with a significant $(p<0.001)$ decrease in antioxidant defense markers (TAC and SOD) in control diabetic group compared with those of normal control group. Treatment with insulin or CQC reversed significantly $(p<0.001)$ all these markers toward normal values; however, the effects of CQC and insulin were nearly analogous to each other. Instead, there were no significant changes $(p>0.05)$ detected in serum levels of MDA or NO or TAC in the normal group administered with CQC when compared with the normal control group except the slight significant reduction $(p<0.01)$ detected for SOD.

\section{Histopathological analysis}

As shown in Figure 2 and Table 4, the pancreas of normal rats treated with CQC showed normal islets of Langerhans with normal $\beta$-cells. On the other hand, the diabetic control rat pancreas tissues have shrunken islets of Langerhans with degeneration and necrosis of cell components. On the contrary, the pancreas of diabetic rats administered with insulin showed normal islets of Langerhans with normal $\beta$-cells. The pancreas of diabetic rat administered with $\mathrm{CQC}$ showed mild atrophy of islets of Langerhans with normal $\beta$-cells.

Table 3. Biochemical parameters of rats' groups.

\begin{tabular}{|c|c|c|c|c|c|}
\hline Groups & Normal control & Normal + CQC & Diabetic control & Diabetic + insulin & Diabetic + CQC \\
\hline \multicolumn{6}{|l|}{ Liver function tests } \\
\hline ALT (IU/l) & $66.2 \pm 5.359$ & $88.9 \pm 3.956^{* * * *}$ & $104.6 \pm 13.910^{* * * *}$ & $77.8 \pm 9.206^{s \$ s}$ & $80.59 \pm 4.288^{\$ \$ \$, *}$ \\
\hline AST (IU/l) & $89.81 \pm 3.173$ & $109.45 \pm 7.430^{* * * *}$ & $144.71 \pm 8.767^{* * * *}$ & $102.01 \pm 6.473^{\$ \$ \$, * *}$ & $91.88 \pm 3.366^{\mathrm{sS}}$ \\
\hline Albumin (g/dl) & $1.85 \pm 0.095$ & $1.36 \pm 0.048^{* * * *}$ & $1.13 \pm 0.061^{* * * *}$ & $1.55 \pm 0.061^{\$ \$ \$, * * *}$ & $1.67 \pm 0.049^{\$ \$ \$, * * *}$ \\
\hline \multicolumn{6}{|l|}{ Kidney function tests } \\
\hline Creatinine (IU/l) & $0.303 \pm 0.019$ & $0.318 \pm 0.027$ & $0.439 \pm 0.0593^{* * * *}$ & $0.289 \pm 0.033^{s s s}$ & $0.379 \pm 0.059^{s, * *}$ \\
\hline Urea $(\mathrm{mg} / \mathrm{dl})$ & $18.89 \pm 1.214$ & $14.76 \pm 1.105$ & $33.05 \pm 2.079^{* * *}$ & $23.88 \pm 2.437^{s s s}$ & $21.96 \pm 1.318^{\$ \$ s}$ \\
\hline Uric acid (mg/dl) & $1.15 \pm 0.148$ & $1.41 \pm 0.229$ & $2.73 \pm 0.833^{* * * *}$ & $1.642 \pm 0.245^{\$ \$, * * *}$ & $1.735 \pm 0.161^{\$ \$, * * * *}$ \\
\hline \multicolumn{6}{|l|}{ Lipid profile } \\
\hline Cholesterol (mg/dl) & $50.41 \pm 2.866$ & $54.76 \pm 3.365$ & $74.38 \pm 7.86^{* * * *}$ & $65.82 \pm 2.524^{\$ \$, * * *}$ & $51.73 \pm 2.728^{s \$ s}$ \\
\hline $\mathrm{TG}(\mathrm{mg} / \mathrm{dl})$ & $46.68 \pm 9.503$ & $56.37 \pm 3.360^{*}$ & $81.59 \pm 6.823^{* * *}$ & $56.97 \pm 6.758^{s s s, *}$ & $70.24 \pm 4.812^{\$, * * *}$ \\
\hline HDL (mg/dl) & $25.96 \pm 3.045$ & $24.84 \pm 3.305$ & $10.50 \pm 2.166^{* * * *}$ & $25.77 \pm 2.279^{s s s}$ & $14.356 \pm 3.040^{\$ S, * * * *}$ \\
\hline LDL (mg/dl) & $15.08 \pm 2.440$ & $18.3 \pm 4.650$ & $47.16 \pm 8.930^{* * * *}$ & $29.10 \pm 3.645^{\$ s \$, * * *}$ & $23.28 \pm 4.970^{\$ \$ \$, *}$ \\
\hline \multicolumn{6}{|c|}{ Lipid peroxidation and antioxidants } \\
\hline $\operatorname{MDA}(\mathrm{nmol} / 1)$ & $1.47 \pm 0.093$ & $2.01 \pm 0.549$ & $9.84 \pm 1.207^{* * * *}$ & $6.26 \pm 1.080^{\$ \$ \$, * * *}$ & $6.11 \pm 0.858^{s \$ s, * * *}$ \\
\hline $\mathrm{TAC}(\mathrm{mmol} / \mathrm{l})$ & $1.63 \pm 0.057$ & $1.506 \pm 0.135$ & $0.604 \pm 0.175^{* * *}$ & $1.58 \pm 0.054^{\$ \$ \$}$ & $0.94 \pm 0.154^{\$ \$ \$, * * *}$ \\
\hline $\mathrm{NO}(\mathrm{mmol} / \mathrm{l})$ & $0.015 \pm 0.0005$ & $0.016 \pm 0.0006$ & $0.042 \pm 0.003^{* * * *}$ & $0.022 \pm 0.002^{\$ \$ \$, * * *}$ & $0.022 \pm 0.003^{\$ \$ \$, * * *}$ \\
\hline $\mathrm{SOD}(\mathrm{U} / \mathrm{ml})$ & $283.0 \pm 4.517$ & $246.3 \pm 8.808^{* *}$ & $50.06 \pm 3.588^{* * *}$ & $170.3 \pm 12.105^{s s s, * * *}$ & $160.08 \pm 3.12^{\$ \$ \$, * * *}$ \\
\hline \multicolumn{6}{|c|}{ Blood glycosylated hemoglobin } \\
\hline $\mathrm{HbA} 1 \mathrm{C}(\%)$ & $2.92 \pm 0.126$ & $2.934 \pm 0.168$ & $4.03 \pm 0.286^{* * *}$ & $3.25 \pm 0.184^{s s s}$ & $2.954 \pm 0.179^{\$ \$ \$}$ \\
\hline
\end{tabular}

Values are introduced as mean $\pm \mathrm{SD}(n=7$ rats/group). CQC $=$ cobalt-quercetin complex.

${ }^{*} p<0.05,{ }^{* *} p<0.01$, and ${ }^{* * * *} p<0.001$, respectively, against normal group. ${ }^{\mathrm{s}} p<0.05,{ }^{\mathrm{s}} p<0.01$, and ${ }^{\$ \$ \$} p<0.001$, respectively, against diabetic group. 


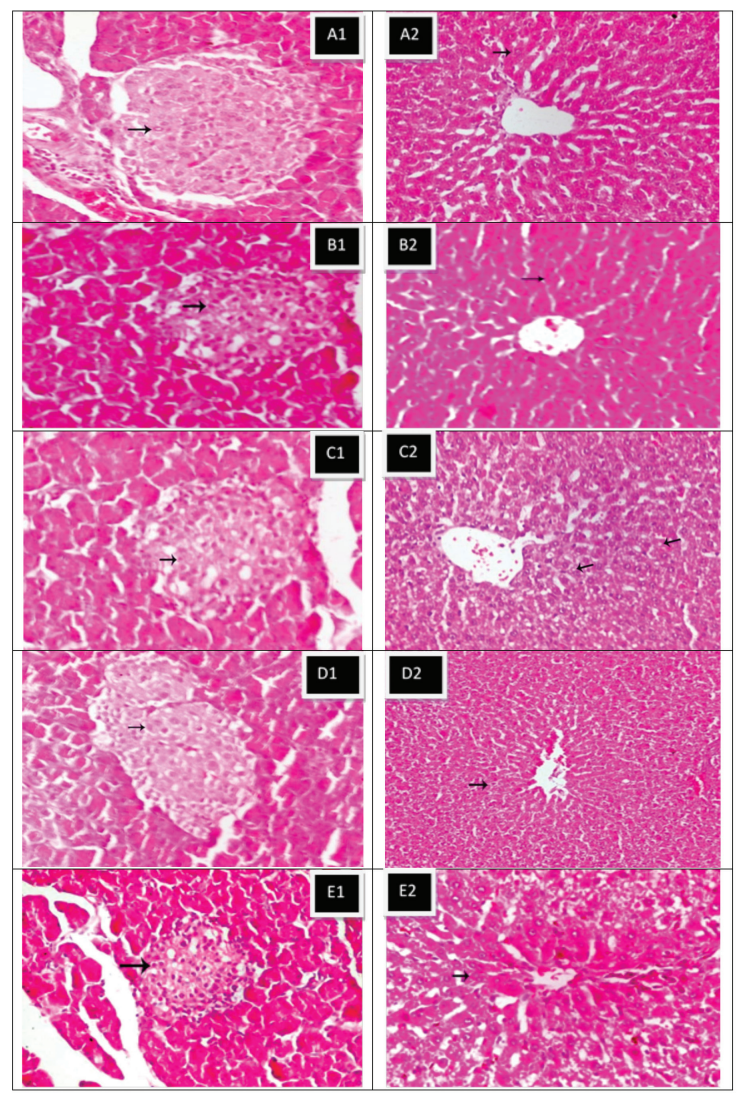

Figure 2. Pancreatic and hepatic tissues histopathology. Pancreas: (A1) normal control rat pancreas showing normal islets of Langerhans with pale rounded and ovoid $\beta$-cells in the center (arrow), embedded in an exocrine portion of the pancreas, (B1) pancreas of normal rat treated with CQC showing normal islets of Langerhans (arrow), (C1) diabetic control rat pancreas showing shrinkage of islets of Langerhans with degeneration and necrosis of cells where its nucleus appeared densely basophilic and karyolysis is evident (arrow), (D1) pancreas of diabetic rat treated with insulin showing normal islets of Langerhans with its normal pale large round- to ovoid-shaped containing cells (arrow) embedded in an exocrine portion of the pancreas, and (E1) pancreas of diabetic rat treated with CQC showing mild atrophy of islets of Langerhans with normal $\beta$-cells (arrow) (H\&E, $\times 400)$. Liver: (A2) normal control rat liver showing normal hepatocytes (arrow) with normal radial arrangements around hepatic cords $(\mathrm{H} \& \mathrm{E}, \times 400)$, (B2) liver of normal rat treated with CQC showing normal hepatocytes (arrow) and normal histological architecture $(\mathrm{H} \& \mathrm{E}, \times 400),(\mathrm{C} 2)$ diabetic control rat liver showing increased vacuolation in the cytoplasm of hepatocytes which appeared as indistinct clear vacuoles (arrows) indicating glycogen infiltration in diabetes $(H \& E, \times 400)$, (D2) liver of diabetic rat treated with insulin showing normal hepatocytes and normal hepatic architecture with mild vacuolation of hepatocytes (arrow) (H\&E, $\times 100)$, and (E2) liver of diabetic rat treated with CQC showing the presence of duplicates indicating regeneration capacity (arrow) with mild vacuolation of hepatocytes $(\mathrm{H} \& \mathrm{E}, \times 100)$.

As shown in Figure 2, the liver section of a normal rat treated with $\mathrm{CQC}$ reflected normal hepatocytes and normal histological architecture. The liver of a diabetic rat showed histopathological changes including increased vacuolation in the cytoplasm of hepatocytes appeared as indistinct clear vacuoles indicating glycogen infiltration in diabetes. On the contrary, liver sections of diabetic rats administered with insulin or with CQC showed normal hepatocytes and normal hepatic architecture with mild vacuolation of hepatocytes. Table 4 illustrated that CQC has a stronger curative/protective action toward liver cells of diabetic rats (normal liver architecture, normal hepatocytes, and no necrosis but mild degeneration of hepatocytes); however, the CQC had the advantage of higher hepatocytes regeneration capacity than insulin (normal liver architecture and normal hepatocytes with mild necrosis and mild degeneration associated with moderate regeneration capacity of hepatocytes).

\section{DISCUSSION}

In the present investigation, the normal rats group treated with CQC showed normal pancreas architecture alongside no significant changes, from the first day until the last day of the experiment, in the FBG levels when compared with those of the corresponding normal control rats group. It also showed normal levels of all other studied biochemical parameters except the observed significant increases in TG, AST, and ALT levels accompanied with the observed significant decreases in serum albumin and SOD reflecting the incidence of mild tolerable liver injury that still can be normally and spontaneously reversed as reflected from the histopathological examination of liver sections excised from normal rats treated with $\mathrm{CQC}$; mild degeneration of few numbers of hepatocytes associated with high capacity of regeneration was detected.

Furthermore, the treatment of normal rats with CQC caused a temporary loss of appetite associated with a slightly more need for drinking water resulting in a significant decrease in the food intake during the first 5-10 days of treatment resulting in a bodyweight loss. These effects started to be reversed toward normal spontaneously with time. This was confirmed by the gain in normal bodyweight of this group of rats by the end of the experiment.

From the day 10 of treatment of diabetic rats with either CQC or insulin, the daily water intake was decreased and continued, while the daily food intake was increased and steps forward. Consequently, the treated rats' bodyweights started to increase and continued. However, with CQC treatment, the bodyweight increase was greater and began earlier than in the case of insulin treatment. These findings suggested that CQC is more powerful than insulin in the minimization of IDDM symptoms.

In the current work, the hypoglycemic effect of CQC was obvious from day 7 of treatment and it was more obvious on the last day of treatment suggesting that CQC may have an inhibitory effect on glucose intestinal absorption and/or a stimulatory effect on insulin release may be via promoting $\beta$-cells regeneration and improving their function. This was in accordance with Hii and Howell (1984) and Zafar and Singh (1990). By comparison with insulin, our biochemical analysis results reflected that $\mathrm{CQC}$ and insulin hypoglycemic effects were nearly alike and reduced FBG by $51 \%$ and $53 \%$, respectively. This was compatible with our histopathological analysis data where insulin treatment showed no atrophy of islets of Langerhans with mild degeneration of $\beta$-cells and CQC treatment showed mild atrophy of islets of Langerhans with no degeneration of $\beta$-cells. This introduced the evidence for the pancreatic curative/ protective effect of $\mathrm{CQC}$, which consequently adds another explanation for its hypoglycemic performance and proves its antidiabetic properties. 
Table 4. Semiquantitative analysis of histopathological studies on rat pancreas and on rat liver in rats' groups.

\begin{tabular}{|c|c|c|c|c|c|}
\hline Criteria & Normal control & Normal+ CQC & Diabetic control & Diabetic + insulin & Diabetic + CQC \\
\hline Atrophy of islets of Langerhans & - & - & +++ & - & + \\
\hline Degeneration of $\beta$-cells & - & - & +++ & + & - \\
\hline Necrosis of hepatocytes & - & - & ++ & + & - \\
\hline Degeneration of hepatocytes & - & + & +++ & + & + \\
\hline Hepatic regeneration capacity & - & ++ & - & ++ & +++ \\
\hline
\end{tabular}

$\mathrm{CQC}=$ cobalt-quercetin complex; $-=$ negative $;+=$ mild $;++=$ moderate $;+++=$ severe .

Diabetes is assumed to encourage lipid peroxidation since elevations in FBG permit excess protein glycation. This glycation disrupts cellular functioning and stimulates free radicals' release as by-products, which ultimately can cause cellular damage in different organs, for example, $\beta$-cells in the pancreas (Saad et al., 2017b). CQC had no significant hypoglycemic influence on normoglycemic rats but antagonized the elevation in circulating glucose under the effect of STZ and this result could be attributed to its antioxidative power. It can be suggested that CQC administration is helpful in diminishing circulated glucose levels and encouraging regeneration of the pancreatic islets as proved by the present histopathological examination of pancreas sections, thus exerting its useful antidiabetic influences. This is in accordance with previously reported effects of certain flavonoids (Hii and Howell, 1984; Wolff, 1993) in alloxan-diabetic animals.

Glycated HbA1C was seen to rise in out of control or badly controlled DM (Bunn et al., 1978). It was proven that glycation possibly will motivate reactive oxygen radicals' genesis in the diabetic state, and the $\mathrm{HbA} 1 \mathrm{C}$ measurement is introduced as a member of the biomarkers of oxidative stress level evaluation in DM (Bravi et al., 2006). Hence, the level of HbA1C is supposed to be a highly sensitive indicator of glycemic regulation (Jain et al., 1989). The current investigation demonstrated that CQC has strong hemostatic properties confirmed by its ability to normalize blood $\mathrm{HbA} 1 \mathrm{C}$ reflecting a high degree of metabolic control.

Bukhari et al. (2008) studied CQC antioxidant activity in vitro and reported that $\mathrm{CQC}$ showed high antioxidant activity. Additionally, the current study established that CQC has a strong antioxidant activity in vivo as well. These antioxidant properties were reflected from CQC ability to increase the level of total antioxidants capacity (TAC) and the activity of the antioxidant defense enzyme SOD, to decrease the levels of the oxidative stress biomarkers MDA, an oxygen radical resulting as a final product of lipid peroxidation in oxygen-dependent degeneration of tissue (Saad et al., 2017e) and NO (Prieto et al., 2007; Saad et al., 2017b), and to normalize the level of the oxidative stress biomarker HbA1C (Bravi et al., 2006) in sera of the treated diabetic rats. Accordingly, CQC is able to work as an active oxygen radicals' scavenger and contribute to different vital organs' protection from diabetic-induced damage in animals. Based on these facts and in accordance with Banihani et al. (2013), we support the previously suggested mechanism by which CQC may affect diabetes. It is via lowering oxidative stress and lipid peroxidation. This lowering may take place by direct neutralization of the created active oxygen radicals, inducing metal chelation activity, increasing certain antioxidant enzyme activities, and suppressing or stimulating definite transcriptional factors.
As one of the conditions that can encourage lipid peroxidation diabetes is one of the pathologic conditions that might indirectly cause liver damage (El-Emshaty et al., 2018). In agreement with Al-Rawi (2007a) and Ene et al.'s (2007) studies, our present results revealed significant lowering in ALT and AST activities and a significant increase in blood albumin in diabetic animals administered with CQC when compared to the control untreated diabetic group. Decreases in ALT and AST activities may be due to increased clearance and/or decreased leakage through hepatocytes membranes as a result of CQC's ability to suppress diabetes-induced oxidative stress, as evidenced earlier. Our histopathological studies verified that CQC has a stronger curative/protective action toward liver cells of diabetic rats (normal liver architecture, normal hepatocytes, and no necrosis but mild degeneration of hepatocytes associated with high regeneration capacity) than insulin (normal liver architecture and normal hepatocytes with mild necrosis and mild degeneration associated with moderate regeneration capacity). Therefore, CQC had the advantage of a high regeneration capacity of hepatocytes associated with no necrosis. Moreover, the treatment of diabetic animals by CQC developed significant decreases in circulatory levels of total cholesterol, TG, and LDL accompanied by a significant increase in HDL level. By comparison, the hypolipidemic effects of insulin and CQC were actually comparable. These hepatoprotective and hypolipidemic actions of CQC may be attributed to its strong antioxidant properties.

Al-Rawi (2007b) reported that STZ-stimulated DM created notable alterations in both the architecture and performance of kidneys in rats. Serum urea, uric acid, and creatinine were significantly higher. Our current study showed that the CQC administration fairly ameliorates renal dysfunction, induced by diabetes and supported by observed significant decreases in serum creatinine, urea, and uric acid levels that may be attributable to its antioxidant properties.

\section{CONCLUSION}

In conclusion, our histological and biochemical findings revealed a remarkable amelioration of all the studied diabetic-induced abnormalities as a result of treating diabetic animals with CQC probably due to the prevention of depletion of the antioxidants, preventing lipid peroxidation, and promoting regeneration of the pancreatic islets. This reflects its strong antioxidant potency. In general, the results of our present study indicate that the antidiabetic effects of CQC against STZ-induced diabetes in rats were comparable to those of insulin; however, CQC to some extent was better. This warrants studies on CQC antidiabetic effects before its possible human use in the future. 


\section{ACKNOWLEDGMENT}

The authors would like to thank Dr. Maha El-Hagrasy, Chemistry Department, Faculty of Science, Damietta University, Egypt, for her support.

\section{CONFLICT OF INTEREST}

The authors declare that there is no conflict of interest.

\section{FUNDING}

None.

\section{REFERENCES}

Abou-seif MA, Youssef A. Evaluation of some biochemical changes in diabetic patients. Clin Chim Acta, 2004; 346:161-70.

Al-Rawi MM. Effect of Trifolium alexandrinum extracts on the kidneys of diabetic rats: biochemical and histological study. Egypt J Hosp Med, 2007b; 29:537-45.

Al-Rawi MM. Effect of Trifolium $s p$. flowers extract on the status of liver histology of streptozotocin-induced diabetic rats. Saud J Biol Sci, 2007a; 14:21-8.

Attallah AM, El-Far M, Abdel Malak CA, Farid K, Omran MM, Yahya RS, Saad EA, Albannan MS, Attallah AA, El Basuni MA, Ali IS, Abed SB, El Naggar MA. A simple diagnostic index comprising epithelial membrane antigen and fibronectin for hepatocellular carcinoma. Ann Hepatol, 2015; 14:869-80.

Badran A, Rubert RE, Ali MA, Sous ES, Herman WH. Diabetes mellitus in Egypt: risk factors, prevalence and future burden. East Mediterr Health J, 1997; 3(1):144-8.

Banihani S, Swedan S, Alguraan Z. Pomegranate and type 2 diabetes. Nutr Res, 2013; 33(5):341-8.

Bravi MR, Armiento A, Laurenti O, Cassano-Faldetta M, De Luca O, Morettia A, De Mattia G. Insulin decreases intracellular oxidative stress in patient with type 2 diabetes mellitus. Metabolism, 2006; 55(5):6915 .

Bukhari BS, Memon S, Tahir MM, Bhanger MI. Synthesis, characterization and investigation of antioxidant activity of cobalt-quercetin complex. J Mol Struct, 2008; 892:39-46.

Bunn HG, Gabby KH, Gallop PM. The glycosylation of hemoglobin: relevance to diabetes mellitus. Science, 1978; 200:21-7.

Chang EL, Simmers C, Knight DA. Cobalt complexes as antiviral and antibacterial agents. Pharmaceuticals, 2010; 3(6):1711-28.

El-Aassar MR, Saad EA, Habib SA, Waly HM. Loading of some

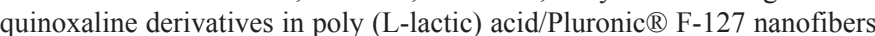
enhances their anticancer efficiency and induces a p53 and p21 apoptoticsignaling pathway. Colloids Surf B Biointerfaces, 2019; 183C:110444; doi:10.1016/j.colsurfb.2019.110444

El-Emshaty HM, Saad EA, Gouida MS, Elshahawy ZR. Associations between CD133, CK19 and G2/M in cirrhotic HCV (genotype-4) patients with or without accompanying tumor. Biocell, 2018; 42(2):55-60.

Ene A, Nwanko E, Samdi L. Alloxan-induced diabetes in rats and effects of black caraway (Carum carvi L.) oil on their body weight. Res J Med Med Sci, 2007; 2(2):48-52.

Elsayed SA, Saad EA, Mostafa SI. Development of new potential anticancer metal complexes derived from 2-hydrazinobenzothiazole. Mini Rev Med Chem, 2019; 19(11):913-22. doi: 10.2174/13895575186661810 17143548

Habib SA, Saad EA, Al-Mutairi FM, Alalawy AI, Sayed MH, El-Sadda RR. Up-regulation of antioxidant status in chronic renal failure rats treated with mesenchymal stem cells and hematopoietic stem cells. Pak J Biol Sci, 2020; 23(6):820-8; doi: 10.3923/pjbs.2020.820.828
Habib SA, Saad EA, Elsharkawy AA, Attia ZR. Pro-inflammatory adipocytokines, oxidative stress, insulin, $\mathrm{Zn}$ and $\mathrm{Cu}$ : interrelations with obesity in Egyptian non-diabetic obese children and adolescents. Adv Med Sci, 2015; 60(2):179-85; doi:10.1016/j.advms.2015.02.002.

Hii C, Howell S. Effects of epicatechin on rat islets of Langerhans. Diabetes, 1984; 33:291-6.

Iacopini P, Baldi M, Storchi P, Sebastiani L. Catechin, epicatechin, quercetin, rutin and resveratrol in red grape: content, in vitro antioxidant activity and interactions. J Food Compost Anal, 2008; 21(8):589-98; doi: 10.1016/j.jfca.2008.03.011

Jain SK, Robert M, John JH. Erythrocyte membrane lipid peroxidation and glycosylated hemoglobin in diabetes. Diabetes, 1989; 38:1539-43.

Mehta JL, Rasouli N, Sinha AK, Molavi B. Oxidative stress in diabetes: a mechanistic overview of its effects on atherogenesis and myocardial dysfunction. Int J Biochem Cell Biol, 2006; 38:794-803.

Naggar El, Bartosikova L, Zemlicka M, Svajdlenka E, Rabiskova M, Strnadova V. Antidiabetic effect of Cleome droserifolia aerial parts: Lipid peroxidation-induced oxidative stress in diabetic rats. Acta Vet Brno, 2005; 74:347-52.

Patil VS, Gutierrez AM, Sunkara M, Morris AJ, Hilt JZ, Kalika DS, Dziubla TD. Curcumin acrylation for biological and environmental applications. J Nat Prod, 2017; 80(7):1964-71.

Prieto JM, Iacopini P, Cioni P, Chericoni S. In vitro activity of the essential oils of Origanum vulgare, Satureja montana and their main constituents in peroxynitrite-induced oxidative processes. Food Chem, 2007; 104(3):889-95.

Ross JA, Kasum CM. Dietary flavonoids: bioavailability, metabolic effects, and safety. Ann Rev Nutr, 2002; 22:19-34.

Saad EA, EL-Demerdash RS, Abd EI-Fattah ME. Mesenchymal stem cells are more effective than captopril in reverting cisplatin-induced nephropathy. Biocell, 2019a; 43(2):73-9.

Saad EA, El-Gayar HA, El-Demerdash RS, Radwan KH Frankincense administration antagonizes adenine-induced chronic rena failure in rats. Pharmacogn Mag, 2018a; 14:634-40.

Saad EA, El-Gayar HA, El-Demerdash RS, Radwan KH. Hepatotoxic risk of gum Arabic during adenine-induced renal toxicity prevention. J Appl Pharm Sci, 2018b; 8(12):104-11; doi: 10.7324/JAPS.2018.8801.

Saad EA, Elsayed SA, Hassanien MM, AL-Adl MS. The new iron(III) 3-oxo-N-(pyridin-2-yl)butanamide complex promotes ehrlich solid tumor regression in mice via induction of apoptosis. Appl Organomet Chem, 2020a; 34(1):e5282; doi:10.1002/aoc.5282

Saad EA, Habib SA, Eltabeey M. Diagnostic performance of AFP, autotoxin and collagen IV and their combinations for non-invasive assessment of hepatic fibrosis staging in liver fibrosis patients associated with chronic HCV. Int J Pharm Qual Assur, 2017a; 8:165-73.

SaadEA, Habib SA, Refai WA, ElfayoumyAA. Malondialdehyde, adiponectin, nitric oxide, C-reactive protein, tumor necrosis factor-alpha and insulin resistance relationships and inter-relationships in type 2 diabetes early stage. Is metformin alone adequate in this stage? Int J Pharm Pharm Sci, 2017b; 9:176-81.

Saad EA, Hassanien MM, El-hagrasy MA, Radwan KH. Antidiabetic, hypolipidemic and antioxidant activities and protective effects of Punica granatum peels powder against pancreatic and hepatic tissues injuries in streptozotocin induced IDDM in rats. Int J Pharm Pharm Sci, 2015b; 7(7):397-402.

Saad EA, Hassanien MM, El-Lban FW. Nickel(II) diacetyl monoxime-2-pyridyl hydrazone complex can inhibit Ehrlich solid tumor growth in mice: a potential new antitumor drug. Biochem Biophys Res Commun, 2017d; 484:579-85.

Saad EA, Hassanien MM, El-mezayen HA, ELmenawy NM. Regression of murine Ehrlich ascites carcinoma using synthesized cobalt complex. Med Chem Commun, 2017c; 8:1103-11. 
Saad EA, Hassanien MM, Elneely EA. Iron(III) diacetylmonoxime-2-hydrazinopyridine complex: a new prospective antitumor drug. Appl Organomet Chem, 2017e; 31:e3684; doi:10.1002/ aoc.3684.

Saad EA, Kiwan HA, Hassanien MM, Al-Adl HE. Synthesis, characterization, and antitumor activity of a new iron-rifampicin complex: a novel prospective antitumor drug. J Drug Deliv Sci Technol, 2020b; 57(C):101671; doi:10.1016/j.jddst.2020.101671

Saad EA, Toson EA, Ahmed GM. Clove or green tea administration antagonizes khat hepatotoxicity in rats. Int J Pharm Pharm Sci, 2015a; 7(6):72-6.

Schellekens AP, Sanders GT, Thornton W, Van Groenstein T. Sources of variation in the column chromatographic determination of glycohemoglobin HbA1. Clin Chem, 1981; 27:94-9.

Shaikh H, Shrivastava VK. Effects of steptozotocin induced diabetes mellitus type 1 on the rat brain antioxidant status and activity of acetyl-cholinesterase: a novel and potential treatment by Vitex negundo. Int J Pharm Pharm Sci, 2014; 6(10):252-6.

Shoskes DA, Nickel JC. Quercetin for chronic prostatitis/chronic pelvic pain syndrome. Urol Clin North Am, 2011; 38:279-84.

Wild S, Roglic G, Green A, Sicree R, King H. Global prevalence of diabetes. Diabetes Care, 2004; 27(5):1047-53.
Wolff S. Diabetes mellitus and free radical. Free radicals, transition metals and oxidative stress in the aetiology of diabetes mellitus and complications. Br Med Bull, 1993; 49:643-9.

Zafar R, Singh J. Antidiabetic activity of Punica granatum Linn. Sci Cult, 1990; 56(7):3.

Zahran F, Saad EA, El-Ablack FZ, Abo Eleneen AM. New synthetic flavonoid with in vitro antitumor activity. J Curr Pharma Res, 2019; 10(1):606-9.

How to cite this article:

Hassanien MM, Saad EA, Radwan KH. Antidiabetic Activity of cobalt-quercetin complex: A new potential candidate for diabetes treatment. J Appl Pharm Sci, 2020; 10(12):044-052. 